\title{
Pointing errors in solar absorption spectrometry - correction scheme and its validation
}

\author{
A. Reichert, P. Hausmann, and R. Sussmann \\ Karlsruhe Institute of Technology, IMK-IFU, Garmisch-Partenkirchen, Germany \\ Correspondence to: A. Reichert (andreas.reichert2@kit.edu) \\ Received: 5 May 2015 - Published in Atmos. Meas. Tech. Discuss.: 22 June 2015 \\ Revised: 20 August 2015 - Accepted: 31 August 2015 - Published: 16 September 2015
}

\begin{abstract}
A method for quantification of sun-pointing inaccuracies in solar absorption spectrometry is presented along with a correction scheme for the resulting errors in trace gas vertical column or profile retrievals. A posteriori correction of pointing errors requires knowledge of both coordinates of the mispointing vector on the solar disk. In principle, quantitative information on the mispointing can be retrieved from Doppler shifts of solar lines derived from measured spectra. However, this yields only one component of the mispointing vector, namely the one which is perpendicular to the solar rotation axis. Missing information on the second vector component has hindered a posteriori correction of mispointing errors so far. Our idea of how to overcome this problem is to obtain estimates of both coordinates of the mispointing by combining subsequent measurements with differing orientations of the solar rotation axis relative to the zenith direction. The proposed concept is suitable in the case of systematic mispointing, i.e., if the mispointing is approximately constant within a given set of measurements. An implementation of this original concept is demonstrated using measurements from the solar absorption Fourier transform infrared (FTIR) spectrometer at the Zugspitze $\left(47.42^{\circ} \mathrm{N}\right.$, $10.98^{\circ}$ E, 2964 ma.s.1.). Soundings in the September 2012 September 2014 time interval were impacted by mispointing problems due to a non-optimum solar tracking optics configuration. They show a mean mispointing in the zenith direction of $-0.063^{\circ}$. This causes biases in vertical soundings of trace gases, e.g., $-2.82 \mathrm{ppb}$ in monthly means of dry-air column-averaged mole fractions of methane $\left(\mathrm{XCH}_{4}\right)$. Measurements made with the more stable pre-September 2012 and post-September 2014 optics configurations show considerably smaller mispointing effects. Applying the mispointing correction, the April 2006-March $2014 \mathrm{XCH}_{4}$ trend deter-
\end{abstract}

mined from Zugspitze measurements is reduced from 6.45 $[5.84,7.04]$ to $6.07[5.55,6.59] \mathrm{ppbyr}^{-1}$. The correction thereby restores consistency with results from the nearby Garmisch FTIR site $\left(47.48^{\circ} \mathrm{N}, 11.06^{\circ} \mathrm{E}, 743\right.$ ma.s.l.). The mispointing correction is applicable to solar absorption measurements in the mid-infrared and near infrared. It will be of particular benefit for refining existing records of highaccuracy-and-precision greenhouse gas soundings for the purpose of improved trend analysis or source-sink inversions.

\section{Introduction}

Solar Fourier transform infrared (FTIR) spectrometry is one of the key measurement techniques for highly accurate ground-based vertical soundings of atmospheric trace gases. Measurements of a large number of atmospheric species have been performed for about 2 decades within the Network for the Detection of Atmospheric Composition Change (NDACC, http://www.ndacc.org) using solar FTIR measurements in the mid-infrared (MIR) spectral range. Measured parameters involve total column amounts of atmospheric species, such as ozone (e.g., Rinsland et al., 1996; Schneider and Hase, 2008); chlorine species (Liu et al., 1992; Rinsland et al., 2003; Kohlhepp et al., 2012; Mahieu et al., 2014); fluorine species (Zander et al., 1987); or climate gases like $\mathrm{N}_{2} \mathrm{O}$ (Zander et al., 1994; Sussmann and Schäfer, 1997), water vapor (e.g., Sussmann et al., 2009; Palm et al., 2010), or methane (e.g., Sussmann et al., 2011, 2012, 2013; Sepúlveda et al., 2012; Ostler et al., 2014, 2015). Additionally, lowresolution vertical profile retrievals have been shown, e.g., for ozone (Pougatchev et al., 1996; Vigouroux et al., 2008), 
carbon monoxide (Pougatchev and Rinsland, 1995; Borsdorff and Sussmann, 2009), and water vapor (e.g., Schneider et al., 2006). More recently, these measurements have been supplemented by high-accuracy-and-precision retrievals of climate gases from the Total Carbon Column Observing Network (TCCON, http://www.tccon.caltech.edu), which relies on solar absorption spectra in the near infrared (NIR) (e.g., Washenfelder et al., 2003; Wunch et al., 2011).

Since solar absorption spectrometers use the sun as a light source, all such instruments contain a device for active tracking of the solar position. The simplest approach to achieve continuous sun tracking is to adjust the observed sky position according to precalculated values. However, such passive tracking does not fulfill typical accuracy requirements. Therefore, most current solar FTIR systems make use of feedback from a quadrant diode to control the solar tracker position and thereby achieve improved pointing accuracy (see, e.g., Adrian et al., 1994; Notholt et al., 1995; Washenfelder et al., 2006). Further improvements can be made by using feedback from a camera image of the position of the solar disc on the spectrometer's entrance aperture instead of a quadrant diode (Gisi et al., 2011). However, the continuous alignment of the instrument's line of sight with the center of the solar disc is generally prone to inaccuracies. Tracking errors in the vertical direction cause the air mass observed by the spectrometer to differ from the air mass assumed in trace gas retrievals (Hase, 2000). To a good approximation, this air mass error results in an equal relative error in the retrieved trace gas columns. Additionally, mispointing may result in further errors in retrieved column amounts, such as instrumental line shape variations if field stops inside the spectrometer are not fully illuminated.

As outlined in Hase (2000), a mispointing of $0.1^{\circ}$ in the zenith direction results in about $1 \%$ trace gas column error for an apparent solar zenith angle (sza) of $80^{\circ}$. These errors exceed current accuracy requirements for measured column amounts, e.g., the $0.5 \%$ accuracy requested for the TCCON network (Toon et al., 2009). It is therefore an issue to determine and correct for the influence of mispointing in solar FTIR measurements.

Information on the mispointing is contained in solar lines which appear in ground-based solar spectra in addition to the terrestrial absorption features. Mispointing perpendicular to the solar rotation axis causes rotational Doppler shifts in the solar lines. Measurements of the solar line shift therefore enable the determination of mispointing perpendicular to the solar rotation axis (Gisi et al., 2011). However, these measurements do not permit complete determination of the mispointing direction and norm since they do not contain information on the mispointing component parallel to the solar rotation axis. This is the basic reason why there is currently no method available that enables a correction of mispointinginduced retrieval errors. In this study, we present a method to overcome this limitation and thereby enable a posteriori cor- rection of mispointing effects, to our knowledge for the first time.

This paper is structured as follows: in Sect. 2, we formulate the mispointing problem and outline the basic idea for its correction. Section 3 gives a detailed description of the mispointing determination scheme and the subsequent trace gas column correction. Section 4 contains an example of the implementation of the correction procedure using FTIR measurements at the Zugspitze $\left(47.42^{\circ} \mathrm{N}, 10.98^{\circ} \mathrm{E}\right.$, 2964 ma.s.1.). Finally the correction results are validated via $\mathrm{XCH}_{4}$ bias and trend analysis using reference solar FTIR measurements at the nearby Garmisch site $\left(47.48^{\circ} \mathrm{N}\right.$, $11.06^{\circ}$ E, 743 ma.s.l.). In Sect. 5 we summarize the results along with some concluding remarks.

\section{Formulation of the mispointing problem and basic idea for a correction method}

For all sun-tracking spectrometers, the alignment of the instrument's line of sight with the sky position of the solar disc center is prone to inaccuracies. The zenith direction component of this mispointing causes the air mass assumed in trace gas column retrievals to differ from the real air mass during the measurement. This error in air mass results in an equally large relative error in the retrieved trace gas column.

As outlined in the previous section, only the mispointing perpendicular to the solar rotation axis and not the component parallel to the axis can be deduced from the Doppler shift of solar lines. Therefore, the component of mispointing in the zenith direction that causes bias in the retrieved trace gas columns cannot be deduced directly from such measurements.

However, the orientation of the solar axis relative to the zenith direction varies over time. If the change of the mispointing between two measurements is negligible compared to the magnitude of the mispointing, the changing axis orientation enables the determination of the zenith and horizontal components of mispointing. The mispointing can therefore be fully constrained from a set of shift measurements at times $t_{1}$ and $t_{2}$ with different axis orientations $\alpha_{\text {axis, } 1}$ and $\alpha_{\text {axis, } 2}$ (Fig. 1). In the following, the vector from the center of the solar disc to the actual pointing coordinates is designated as mispointing vector $\boldsymbol{m}$.

Neglecting differential solar rotation (addressed in Sect. 3), i.e., assuming constant angular velocity at all solar latitudes, a single shift measurement constrains the mispointing to lie on the straight line $s$ parallel to the solar rotation axis $a$. The distance between $s$ and $a$ is proportional to the measured solar line shift $\Delta v / v$. Combining two measurements with different axis orientations $\alpha_{\mathrm{axis}, 1}$ and $\alpha_{\mathrm{axis}, 2}$, the mispointing vector $\boldsymbol{m}$ is then defined by the intersection coordinates of the corresponding straight lines $s_{1}$ and $s_{2}$, respectively. In order to reduce errors due to inaccurate solar line shift measurements, it is favorable to combine larger sets of 


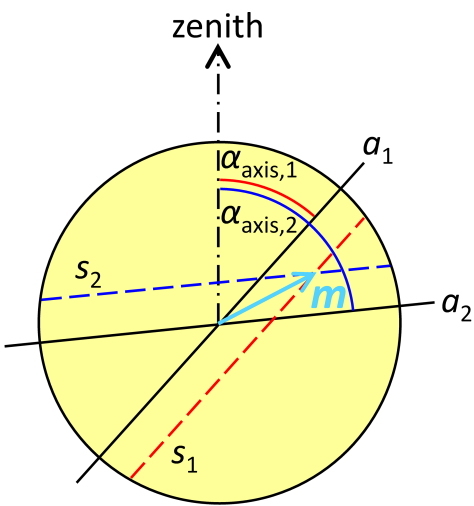

Figure 1. Schematics of the mispointing determination strategy. Solar line shifts are measured at times $t_{1}$ and $t_{2}$ with corresponding solar axis orientations $\alpha_{\text {axis, } 1}$ and $\alpha_{\text {axis, } 2 \text {. The mispointing vector } \boldsymbol{m}}$ is defined by the intersection of the lines $s_{1}$ and $s_{2}$ parallel to the solar rotation axes $a_{1}$ and $a_{2}$, respectively. Differential solar rotation is neglected in this figure.

measured spectra to compute a mean mispointing vector instead of using only two measurements. In this case, the mean mispointing is defined by the mean intersection coordinates of all pairs of measurements that can be selected from a given set of spectra. Note that this approach relies on the assumption that changes of the mispointing within the set of spectra used to compute one mean value of $\boldsymbol{m}$ are negligible compared to the magnitude of the mispointing itself.

\section{Detailed implementation of the mispointing quantification and correction}

In the following, the steps required to implement the mispointing determination approach are described in detail. As a first step, the time series of measured spectra is divided in time bins. We outlined in the previous section that the mispointing determination method relies on the assumption of approximately constant mispointing within each of the time bins. Therefore, the suitable choice of time bins results in a trade-off between low statistical errors in mispointing results achieved by a large bin size and improved fulfillment of the underlying assumption of constant mispointing within each time bin that results from a smaller bin size. An example for the bin size adjustment in the case of the Zugspitze measurements is discussed in Sect. 4.2.

For all measured spectra, the solar line Doppler shift $\Delta v / v$ has to be determined. In our analysis, this task is performed using the PROFFIT software (Hase et al., 2004). The fit is performed on spectra measured with the optical bandpass filter used for $\mathrm{CH}_{4}$ column retrievals, which covers the wave number range $2400<v<3100 \mathrm{~cm}^{-1}$. In order to assess the accuracy of the solar line shift fit, we compare these results to solar line shift measurements in the adjacent $2000<v<$ $2650 \mathrm{~cm}^{-1}$ filter. The differences between the measured line shifts in both filters were set as an estimate of the shift measurement uncertainty. This uncertainty is subsequently used for the calculation of the mispointing uncertainty (see Appendix B).

In addition to solar line shifts, the mispointing calculation requires knowledge of the orientation $\alpha_{\text {axis }}$ of the solar rotation axis relative to the zenith direction for each measured spectrum. More precisely, the orientation angle used in the mispointing calculation is the apparent angle from zenith direction to sun rotation axis direction at the time and location of the measurements, i.e., the angle between projections of zenith direction and sun rotation axis direction on a plane perpendicular to spectrometer's line of sight. All necessary steps for calculation of $\alpha_{\text {axis }}$ are outlined in Appendix A.

Systematic mispointing is assumed to be caused by deviations of the sun tracker optics geometry from an idealized setup. Possible scenarios include, e.g., inaccuracies in mirror orientation or misalignment of the quadrant diode that allows continuous adjustment of the instrument's line of sight to follow the sun position. For such sources of tracking error, it is plausible to assume constant mispointing during limited time intervals in a coordinate system valid in the spectrometer, contrary to, e.g., the azimuth/elevation coordinate system in the sky. Due to the sun tracker optics, the image of the sun undergoes reflections and rotations when transferred to the spectrometer entrance aperture. Therefore, the resulting image of the sun on the entrance aperture has to be calculated for each measurement.

The transformation of the solar image due to the solar tracker optics can be described by a concatenation of $3 \times 3$ imaging matrices resulting in an overall transfer matrix $\mathbf{T}$. The orientation of the sun rotation axis on the entrance aperture is then calculated by multiplying a vector with orientation $\alpha_{\text {axis }}$ with the correct transfer matrix T. For the retransformation of a mispointing vector $\boldsymbol{m}$ derived in spectrometer coordinates to sky coordinates $\boldsymbol{m}_{\mathrm{sky}}, \boldsymbol{m}$ has to be multiplied with the inverse of the transfer matrix, that is, $\boldsymbol{m}_{\text {sky }}=\mathbf{T}^{-1} \boldsymbol{m}$.

Contrary to the simplified solution scheme presented in Sect. 2, the angular velocity of solar rotation depends on solar latitude $\varphi$, a phenomenon called differential solar rotation. The latitude-dependent angular velocity of solar rotation $\omega(\varphi)$ is described approximately by the following relation:

$\omega(\varphi)=c_{1}+c_{2} \sin ^{2} \varphi+c_{3} \sin ^{4} \varphi$.

Currently accepted values for the constants $c_{1}, c_{2}$, and $c_{3}$ are $c_{1}=14.713 \pm 0.0491^{\circ} \mathrm{d}^{-1}, c_{2}=-2.396 \pm 0.188^{\circ} \mathrm{d}^{-1}$, and $c_{3}=-1.787 \pm 0.253^{\circ} \mathrm{d}^{-1}$ (Snodgrass and Ulrich, 1990). Due to differential solar rotation, a single solar line shift measurement does not constrain the mispointing to lie on a straight line as depicted in Fig. 1, but on a line with a constant velocity component along the observer's line of sight.

The radial mispointing in degree perpendicular to the solar rotation axis $m_{\text {rad }}$ can be calculated from solar line shift 


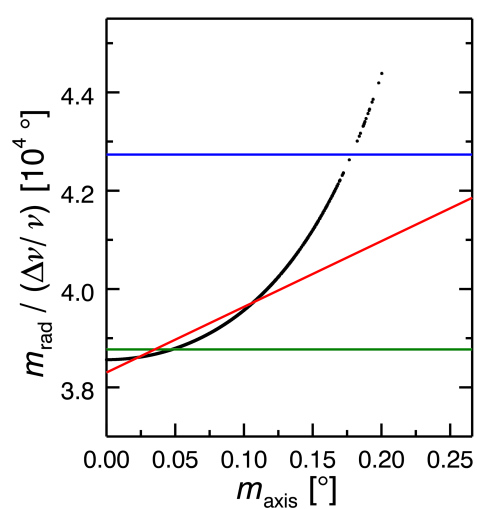

Figure 2. Conversion of solar shift to radial mispointing. $m_{\text {axis }}$ designates the apparent distance from the solar equator. Blue: mean conversion factor given in Gisi et al. (2011). Green: iteratively determined mean conversion for the Zugspitze data set according to Eq. (3). Black: conversion according to Eq. (2), using $\omega(\varphi)$ values calculated with Eq. (1), $m_{\text {axis }}$ calculated using Eq. (3). Red: best fit linear conversion according to Eq. (4).

measurements:

$m_{\mathrm{rad}}=[\Delta v / v \cdot c / \omega(\varphi)] \cdot r_{\mathrm{sun}, \text { apparent }} / r_{\mathrm{sun}}$,

where $c=2.99792458 \times 10^{8} \mathrm{~ms}^{-1}, \quad r_{\text {sun }}=6.958 \times 10^{8} \mathrm{~m}$, and $r_{\text {sun, apparent }}=0.266^{\circ}$.

In this study, instead of using Eq. (2) for mispointing calculation, a linear approximation to this relation is performed (see Fig. 2). Mispointing results determined from Zugspitze measurements suggest this approximation induces no significant additional error in the results. While the mean relative error of mispointing over the Zugspitze measurement time series is $9.5 \%$, the error of radial mispointing induced by the linear approximation is $0.36 \%$, which is negligible compared to the mispointing error.

The following steps are performed to obtain the linear approximation:

i. A mean factor for conversion of solar shifts into mispointing is determined iteratively from our data set. To obtain the conversion factor, the distributions of mispointing values parallel and perpendicular to the solar equator are assumed to be similar. Using the shiftmispointing conversion given in Gisi et al. (2011), a typical distribution of mispointing values parallel to the solar rotation axis is then calculated from the solar line shift measurements. By means of Eq. (1), this mispointing distribution can be converted to a typical angular velocity distribution for the given data set. The mean value of this $\omega(\varphi)$ distribution is then used to calculate an improved shift-mispointing conversion factor replacing $\omega(\varphi)$ in Eq. (2) by the mean value of the angular velocity distribution. The above steps are repeated iteratively until convergence is reached. This results in an

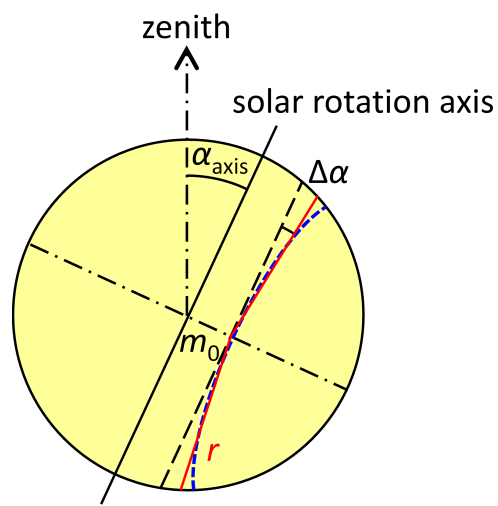

Figure 3. Linear approximation of differential rotation. A solar line shift measurement constrains the mispointing to lie on a line with constant velocity component along the observer's line of sight (blue line). Linear approximation of this relation results in a constraint of mispointing on rays $r$ tilted away from the solar rotation axis by an angle $\Delta \alpha$ and with a minimum distance $m_{0}$ to the axis (red line).

adjusted shift-mispointing conversion of

$m_{\mathrm{rad}, \text { mean }}=\Delta v / v \cdot 38771.4^{\circ}$.

ii. The distribution of mispointing parallel to the solar equator $m_{\text {rad }}$ can now be calculated using Eq. (3). We assume that the distribution of mispointing parallel to the sun rotation axis $m_{\text {axis }}$ is similar to the distribution

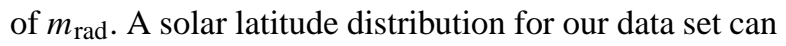
now be derived using $\varphi=\arcsin \left(m_{\text {axis }} / r_{\text {sun, apparent }}\right)$. This enables calculating $\omega(\varphi)$ by means of Eq. (1).

iii. A linear fit to the mispointing values calculated using Eq. (2) and the angular velocity values determined in step (ii) is then performed. The final result for the best fit linear approximation is

$m_{\mathrm{rad}, \text { lin }}=\Delta v / v \cdot\left(a+b \cdot m_{\mathrm{axis}}\right)$

where $a=38301.9 \pm 2.9^{\circ}$ and $b=13363 \pm 63$.

Equation (4) implies that a solar shift measurement constrains the mispointing to lie on a pair of rays $r$ with a distance to the solar rotation axis on the solar equator of $m_{0}=$ $\Delta v / v \cdot a$, and at an angle relative to the zenith direction of $\alpha_{\text {corr }}=\alpha_{\text {axis }} \pm \Delta \alpha$, where $\Delta \alpha=\arctan (\Delta v / v \cdot b)$, as shown in Fig. 3.

Given the orientation of the solar axis and the conversion of solar line shift to mispointing, we can now calculate mispointing results. As described at the beginning of this section, the measurement time series is distributed in time bins. For each bin, all possible combinations of measurement pairs are considered. For each of the $n(n-1) / 2$ possible pairs selected from the $n$ measurements within a time bin, a mispointing vector $\boldsymbol{m}$ is calculated. Finally, the mean of the mispointing over each time bin is calculated as the error-weighted mean 
of all $\boldsymbol{m}$. The computation of the weighted means requires an estimate of the uncertainty of intersection coordinates. The uncertainty estimate was deduced from the difference of coincident solar shift measurements in adjacent optical filters. A detailed description of this uncertainty estimate is given in Appendix B.

Corrected trace gas column amounts can be calculated from the mispointing results with two distinct correction methods: the a posteriori correction method consists in subtracting the mispointing in the zenith direction $m_{y}$,sky from the apparent sza taken from the trace gas retrieval ray trace calculation. Corrected air mass values can then be calculated according to

air mass $_{\mathrm{corr}}=1 / \cos \left(\mathrm{sza}-m_{y, \text { sky }}\right)$.

We then multiply retrieved trace gas columns with air

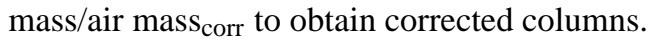

The second (a priori) correction method takes into account that, in addition to the effect on the conversion of slant path to vertical column, the mispointing has slight influence on the forward spectral calculations performed in the retrieval. The a priori method therefore uses the mispointing-corrected sza as an input to the retrieval. The retrieval is then repeated, with the corrected input values resulting in corrected trace gas column results.

Note that Eq. (5) given in the a posteriori method only permits an approximate air mass calculation. In order to achieve higher accuracy especially at high sza, a more sophisticated calculation such as the approach described by Kasten and Young (1989) can be chosen. Furthermore, the a priori scheme includes additional effects such as the influence of mispointing on the ray trace calculation in trace gas retrievals which are not considered in the a posteriori method. However, as shown in Sect. 4.3, the corrected trace gas columns obtained using the a posteriori approach show good consistency with the results from the more sophisticated a priori approach.

\section{Example correction for Zugspitze FTIR}

\subsection{Solar FTIR and tracker setup}

The solar FTIR instrument considered in the example correction is located at the Zugspitze $\left(47.42^{\circ} \mathrm{N}, 10.98^{\circ} \mathrm{E}\right.$, 2964 ma.s.l.). The system is based on a Bruker IFS 125 instrument, and it is described in detail in Sussmann and Schäfer (1997). For the validation of correction results we furthermore use data from the nearby Garmisch FTIR ( $47.48^{\circ} \mathrm{N}, 11.06^{\circ} \mathrm{E}, 743 \mathrm{ma}$ a.s.1.).

The geometry of the Zugspitze sun tracker is depicted schematically in Fig. 4. The custom-made tracker is located above the spectrometer, in an astronomical dome that is opened for measurements. The tracker optics consists of a first plane $45^{\circ}$ elevation mirror $\left(M_{1}\right)$ to adjust the elevation

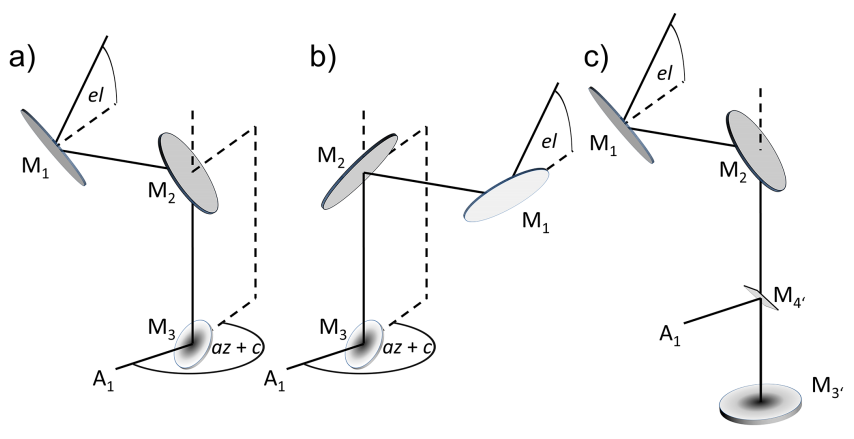

Figure 4. Geometry of the Zugspitze solar tracker optics. (a) Current optical setup after September 2014, Flip 1. (b) Current setup, Flip 0. (c) Optical setup before September 2012.

angle (el) of the instrument's line of sight. Sunlight is then guided to the spectrometer below by a second plane $45^{\circ}$ mirror $\left(\mathrm{M}_{2}\right)$. The azimuth of the line of sight (az) is adjustable by rotation of both mirrors on an arm. The same sky position can be reached by the tracker system with two distinct arm orientations with $180^{\circ}$ position difference: Flip 1, used for most measurements and depicted in Fig. 4a, and Flip 0, used for measurements with az $<116^{\circ}$ or az $>296^{\circ}$ since September 2012; see Fig. 4b.

The setup in place since September 2012 furthermore contains an off-axis mirror $\left(\mathrm{M}_{3}, f=478 \mathrm{~mm}\right)$ that changes beam orientation to horizontal again and creates an image of the sun on the aperture $A_{1}$. The image of the sun is centered on the aperture $A_{1}$ by a quadrant diode continuously giving feedback for tracker positioning. Since September 2014, the active tracking by means of the quadrant diode has been replaced by a Camtracker (Gisi et al., 2011) system inside the spectrometer. Before September 2012, horizontal beam direction and creation of an image on $\mathrm{A}_{1}$ was achieved by the parabolic mirror $\mathbf{M}_{3}^{\prime}(f=1920 \mathrm{~mm})$ and an additional plane mirror $\mathrm{M}_{4}^{\prime}$, instead of the off-axis mirror $M_{3}$ (see Fig. 4c). The optical configuration was changed in September 2012 in order to enable radiometric calibration of the measured spectra by means of a high-temperature blackbody source. However, due to the smaller size of the solar image at $A_{1}$ in the new setup, tracking accuracy has been degraded significantly by the modification. This issue highlights the fact that optimum performance of quadrant-diode-based sun tracker systems is only ensured within a narrow range of optical configurations. Great care has to be taken when changing parameters such as solar image size to maintain tracking accuracy. Camera-based setups such as the Camtracker system are less sensitive to optical system modifications. The installation of such a system therefore enabled the regaining of high tracking accuracy for the Zugspitze FTIR. 


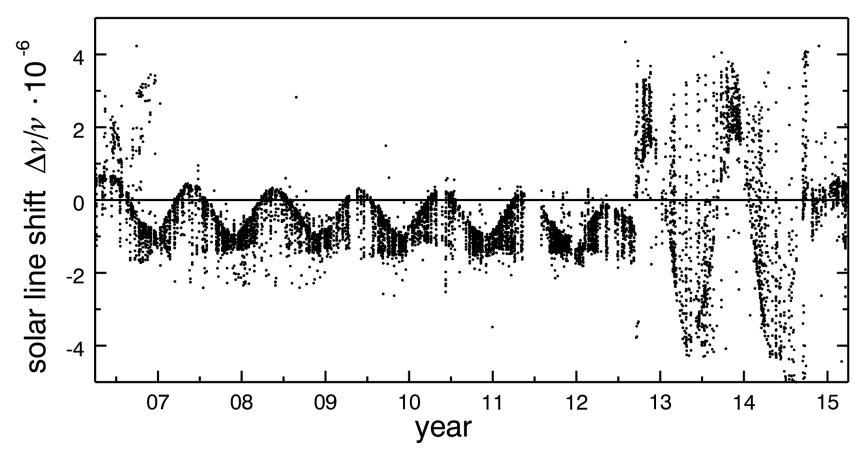

Figure 5. Time series of solar line shifts determined from Zugspitze FTIR spectra in the $2400<v<3100 \mathrm{~cm}^{-1}$ optical filter.

\subsection{Implementation of correction}

The first step in the implementation of the mispointing correction procedure consists in deducing solar line shifts from the measured spectra as described in Sect. 3. Figure 5 shows the time series of solar line shifts obtained from the Zugspitze measurements.

As mentioned in Sect. 3, the transformation of the solar image by the tracker optics can be characterized by $3 \times 3$ imaging matrices that describe changes in beam direction and changes of the solar image in the image plane perpendicular to the beam direction. In the following, we establish those transfer matrices for the optics setup described in Sect. 4.1. The corresponding transfer matrices are established for the configurations Flip 1 and Flip 0 in the current optical setup shown in Fig. $4 a$ and b, and the Flip 1 configuration for the pre-September 2012 setup shown in Fig. 4c.

The complete transformation can be described by a concatenation of three different types of operations: (i) reflections on a plane mirror perpendicular to the beam direction; (ii) rotation of the coordinate system; and, finally, (iii) reflection on $45^{\circ}$ mirrors. Operation (i) corresponds to mirror matrices $\mathbf{M}$, e.g., for a mirror in the $x y$ plane:

$\mathbf{M}_{x y}=\left(\begin{array}{ccc}1 & 0 & 0 \\ 0 & 1 & 0 \\ 0 & 0 & -1\end{array}\right)$.

Operation (ii) is described by the standard $R^{3}$ rotational matrices $\mathbf{R}_{x / y / z, \varphi}$, where $x / y / z$ designates the direction of the rotation axis and $\varphi$ the rotation angle in mathematical orientation. Note that a rotation of the coordinate system by an angle $\varphi$ corresponds to $\mathbf{R}_{x / y / z,-\varphi}$. Reflection on $45^{\circ}$ mirrors (iii) can be further decomposed in a combination of rotation according to (ii) to a coordinate system in which the mirror lies on a plane of base vectors, e.g., the $x y$ plane. This rotation is followed by a reflection according to (i) and a final backwards rotation of the coordinate system. In the following, the transfer matrices for $45^{\circ}$ mirrors are designated $\mathbf{S}_{x / y \pm}$, where the $x / y$ index specifies the coordinate axis parallel to the mirror if the $z$ axis is orientated in beam direction prior to the reflection. The sign in the index is "- " if the third axis points towards the mirror and " + " if the third axis points away from the mirror. The matrices $\mathbf{S}_{x / y \pm}$ have the following form:

$$
\mathbf{S}_{x \pm}=\left(\begin{array}{ccc}
1 & 0 & 0 \\
0 & 0 & \pm 1 \\
0 & \pm 1 & 0
\end{array}\right), \quad \mathbf{S}_{y \pm}=\left(\begin{array}{ccc}
0 & 0 & \pm 1 \\
0 & 1 & 0 \\
\pm 1 & 0 & 0
\end{array}\right)
$$

Using the basic operations (i), (ii), and (iii), we can now deduce transfer matrices $\mathbf{T}$ for the Zugspitze sun tracker system. The initial coordinate system valid in the sky is defined with the $x$ axis in the horizontal direction, the $y$ axis in the zenith direction and the $z$ axis along the line of sight towards the instrument. After each sub-step of the path through the tracker optics, the coordinate system is rotated to orient the $z$ axis in ingoing beam direction.

The individual steps can be tracked in Fig. 4. For the current setup and Flip 1 configuration (Fig. 4a), the transfer matrix components and final result are

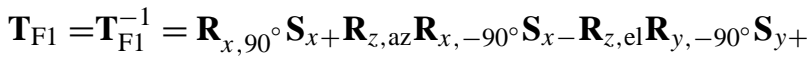

$$
\begin{aligned}
& =\left(\begin{array}{ccc}
-\cos (-\mathrm{az}+\mathrm{el}) & -\sin (-\mathrm{az}+\mathrm{el}) & 0 \\
-\sin (-\mathrm{az}+\mathrm{el}) & \cos (-\mathrm{az}+\mathrm{el}) & 0 \\
0 & 0 & 1
\end{array}\right) \text {. }
\end{aligned}
$$

Note that $\mathbf{T}_{\mathrm{F} 1}$ describes the transfer to a spectrometer coordinate system in which the $x$ and $y$ axes are rotated relative to the horizontal and vertical direction by a constant angle $c$. As visible in Fig. 4, this constant rotation angle $c$ depends on the orientation of the spectrometer and is equal for all measurements in all optical configurations. Since a transformation to a common spectrometer coordinate system with arbitrary orientation is sufficient for our analysis, the constant rotation is neglected in all transfer matrices.

In Flip 0 configuration (Fig. 4b), the corresponding matrix is given by

$$
\begin{aligned}
\mathbf{T}_{\mathrm{F} 0}= & \mathbf{T}_{\mathrm{F} 0}^{-1}=\mathbf{R}_{x, 90^{\circ}} \mathbf{S}_{x+} \mathbf{R}_{z, \mathrm{az}} \mathbf{R}_{z, 180^{\circ}} \mathbf{R}_{x,-90^{\circ}} \\
& \mathbf{S}_{x-} \mathbf{R}_{z,-\mathrm{el}} \mathbf{R}_{y, 90^{\circ} \mathbf{S}_{y-}} \\
= & \left(\begin{array}{ccc}
\cos (\mathrm{az}+\mathrm{el}) & -\sin (\mathrm{az}+\mathrm{el}) & 0 \\
-\sin (\mathrm{az}+\mathrm{el}) & -\cos (\mathrm{az}+\mathrm{el}) & 0 \\
0 & 0 & 1
\end{array}\right) .
\end{aligned}
$$

For the optical setup before September 2012 and the Flip 1 configuration (Fig. 4c), we obtain

$$
\begin{aligned}
\mathbf{T}_{\mathrm{F} 1 \text { old }}= & \mathbf{R}_{x,-90^{\circ}} \mathbf{S}_{x \pm} \mathbf{R}_{y, 180^{\circ}} \mathbf{M}_{x y} \mathbf{R}_{z, \mathrm{az}} \mathbf{R}_{x,-90^{\circ}} \\
& \mathbf{S}_{x-} \mathbf{R}_{z, \mathrm{el}} \mathbf{R}_{y,-90^{\circ}} \mathbf{S}_{y+} \\
= & \left(\begin{array}{ccc}
\cos (-\mathrm{az}+\mathrm{el}) & \sin (-\mathrm{az}+\mathrm{el}) & 0 \\
-\sin (-\mathrm{az}+\mathrm{el}) & \cos (-\mathrm{az}+\mathrm{el}) & 0 \\
0 & 0 & 1
\end{array}\right) \neq \mathbf{T}_{\mathrm{F} 1 \text { old }}^{-1} \\
\mathbf{T}_{\mathrm{F} 1 \text { old }}^{-1}= & \left(\begin{array}{ccc}
\cos (-\mathrm{az}+\mathrm{el}) & -\sin (-\mathrm{az}+\mathrm{el}) & 0 \\
\sin (-\mathrm{az}+\mathrm{el}) & \cos (-\mathrm{az}+\mathrm{el}) & 0 \\
0 & 0 & 1
\end{array}\right)
\end{aligned}
$$




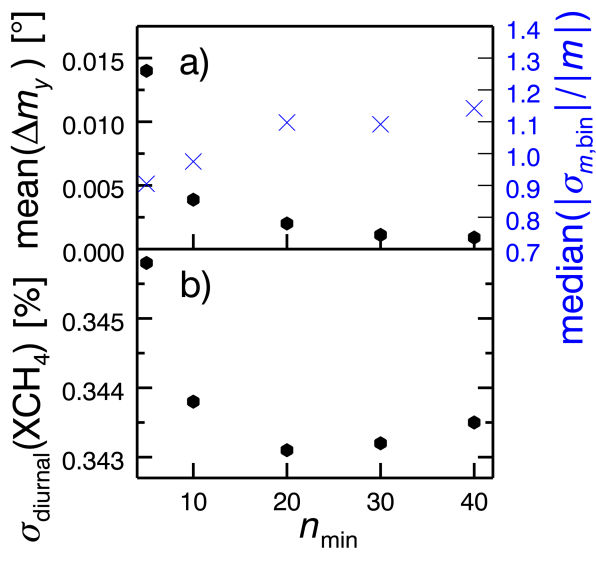

Figure 6. Choice of time bin size for Zugspitze mispointing determination. (a) Mean zenith mispointing error (black) and median of the relative scatter of mispointing results within time bins (blue). (b) Mean $1 \sigma-\mathrm{XCH}_{4}$ diurnal variation.

The next analysis step consists in dividing the measurement time series in time bins. For the Zugspitze measurements, each time bin was chosen to comprise the number of measurement days necessary to reach a total number of at least $n_{\min }$ spectra in the bin. The choice of time bin size $n_{\min }$ results from a trade-off between the statistical uncertainty of the mispointing result and the degree of fulfillment of the assumption that mispointing is approximately constant within each time bin. Figure $6 \mathrm{a}$ demonstrates this trade-off: the mean error of the mispointing result in the zenith direction $\left(\Delta m_{y}\right)$ declines with increasing bin size. Variations of the mispointing within a time bin result in scatter of the intersection coordinates for different measurement pairs included in the bin. Therefore, the scatter of the intersection coordinates as given by their standard deviation divided by the norm of the mispointing vector indicates how well the assumption of constant mispointing is fulfilled. As visible in Fig. 6a, the median of this intra-bin scatter increases with increasing $n_{\min }$, indicating more variability of mispointing within time bins. As an indicator to find an optimum $n_{\min }$, we use the mean of $\mathrm{XCH}_{4}$ diurnal variation over the measurement time series (see Sussmann et al., 2011). Since the underlying real $\mathrm{XCH}_{4}$ diurnal variation can be assumed to be significantly lower than the measured values, $n_{\min }$ is chosen to minimize the measured diurnal variation. As shown in Fig. $6 \mathrm{~b}$, a bin size of $n_{\min }=20$ is chosen for the Zugspitze measurements based on this criterion. The mean number of measurement days included in a time bin is 7.1 days.

\subsection{Results and validation via methane trend analysis}

In this section, the mispointing results based on the Zugspitze measurements and their validation by means of $\mathrm{XCH}_{4}$ trend analysis are presented. We consider the time interval from April 2006 until March 2015. This time interval was cho-
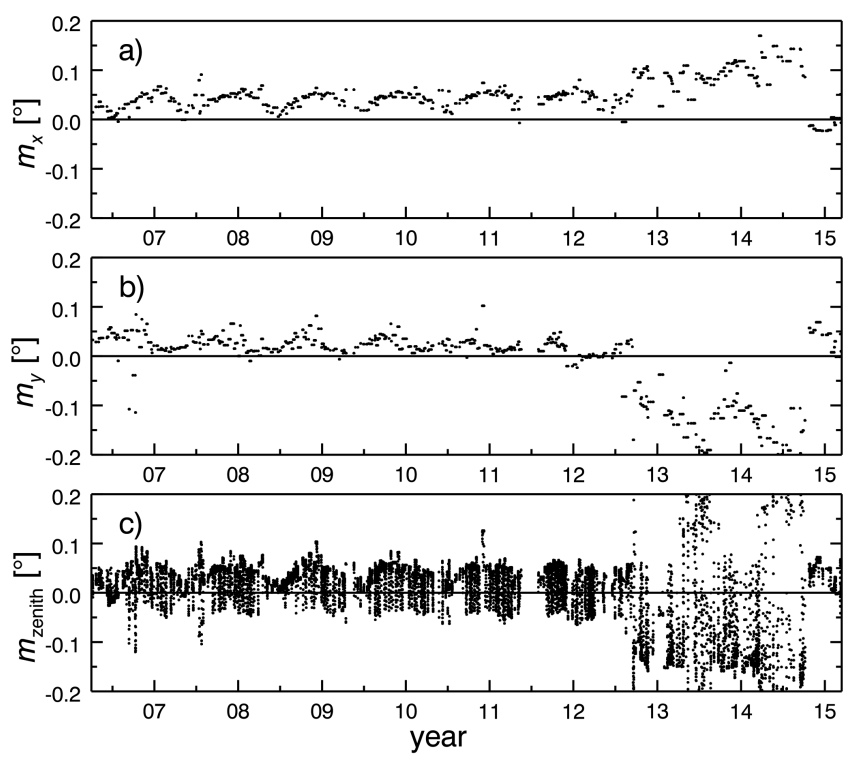

Figure 7. Mispointing results determined from Zugspitze measurements. (a) $x$ component of mispointing in spectrometer coordinates. (b) $y$ component of mispointing in spectrometer coordinates. (c) Zenith component of mispointing in sky coordinates.

sen based on three criteria: it coincides with the phase of renewed increase of atmospheric methane concentrations (e.g Sussmann et al., 2012). Furthermore, the interval includes the most up-to-date measurements available. The last criterion is that the interval length be a multiple of a 1-year period, which is crucial for avoiding bias in the trend analysis.

The time series of mispointing in spectrometer coordinates and the zenith component of the mispointing in sky coordinates are shown in Fig. 7. The mean mispointing in the zenith direction over the measurements made in the April 2006September 2012 time interval, which corresponds to the optics setup shown in Fig. 4c, is $0.024 \pm 0.001^{\circ}$. Within the September 2012-September 2014 interval (optics setup shown in Fig. 4a and b and quadrant diode) the mean mispointing is $-0.063 \pm 0.005^{\circ}$, and for the October 2014March 2015 interval (optics setup shown in Fig. 4a and b and Camtracker) it is $0.024 \pm 0.008^{\circ}$.

The mispointing determination scheme relies on the basic assumption of approximately constant mispointing within each time bin. The validity of this assumption can be evaluated as outlined in Sect. 4.2, namely by analysis of the intrabin scatter of mispointing results. Throughout the September 2012-September 2014 time interval, the median of the scatter of mispointing results within each time bin was only $46 \%$ of the mispointing vector norm in spectrometer coordinates, which means that the assumption of constant mispointing within each time bin is appropriate and leads to a meaningful trace gas column correction. For the April 2006September 2012 and October 2014-December 2014 phases, the intra-bin mispointing scatter corresponds to 108 and 


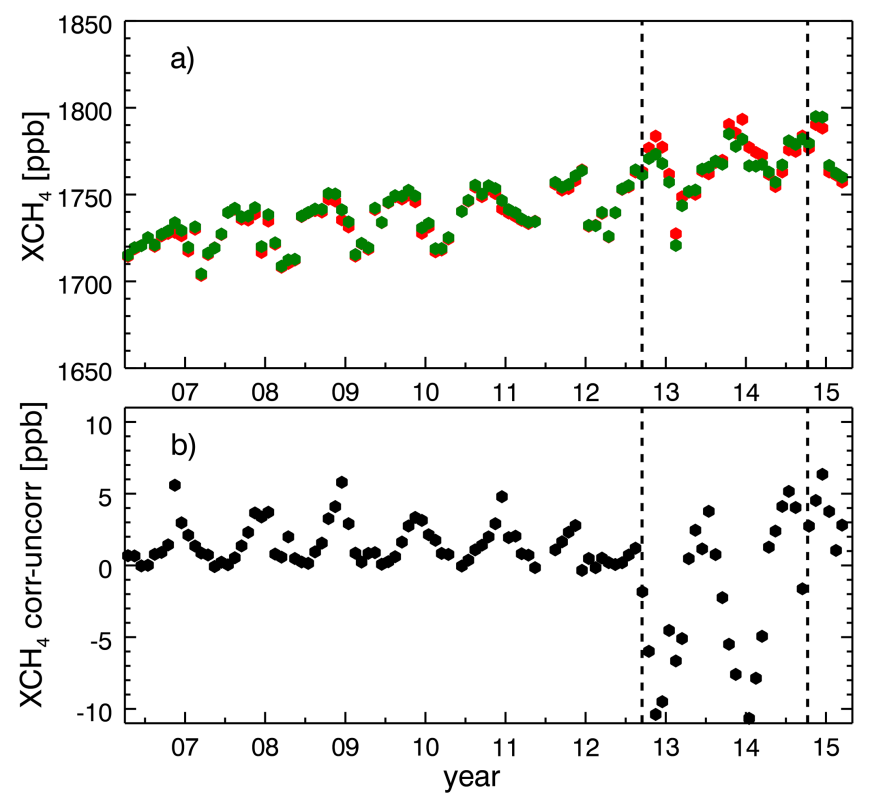

Figure 8. (a) Comparison of a posteriori-corrected (green) and uncorrected (red) Zugspitze $\mathrm{XCH}_{4}$ monthly mean time series. (b) Time series of differences between a posteriori-corrected and uncorrected $\mathrm{XCH}_{4}$ monthly mean time series.

$152 \%$ of the mispointing vector norm in spectrometer coordinates, respectively. Therefore, the basic assumption of constant mispointing is only poorly fulfilled in these phases and in general for the Garmisch instrument. This is due to the fact that the mispointing values in these phases were generally very small, which makes a mispointing determination with small relative error challenging. However, due to the small mispointing values, the mispointing correction has only very minor influence on the $\mathrm{XCH}_{4}$ values in these time intervals.

The use of the a posteriori correction method presented in Sect. 3 instead of the full a priori correction results in a $5 \%$ bias in the zenith component of the mispointing for the Zugspitze measurement time series. This corresponds to only $\sim 0.02 \%$ bias in $\mathrm{XCH}_{4}$. Therefore, the a posteriori method can be considered to provide a fair approximation to the a priori results, which has the advantage of not having to repeat the trace gas retrieval procedure after the mispointing determination.

Figure 8a shows a comparison of the corrected and uncorrected Zugspitze time series of $\mathrm{XCH}_{4}$ monthly means for the phase of renewed methane increase since 2006. In Fig. $8 \mathrm{~b}$, the mispointing correction applied to the original time series is shown. The mean of $\mathrm{XCH}_{4}$ correction over all measurements made in the time interval most affected by the mispointing (September 2012-September 2014) is $-2.82 \pm 2.08 \mathrm{ppb}$.

The mispointing results are validated by means of methane trend analysis. To perform this analysis, monthly means are calculated from the uncorrected and corrected $\mathrm{XCH}_{4}$ time
Table 1. $\mathrm{XCH}_{4}$ trend $\left(\mathrm{ppbyr}^{-1}\right)$ for the April 2006-March 2015 time interval and corresponding $95 \%$ confidence intervals; Zugspitze and Garmisch sites, uncorrected, a posteriori and a priori corrected.

\begin{tabular}{llll}
\hline & uncorrected & $\begin{array}{l}\text { a posteriori } \\
\text { corrected }\end{array}$ & a priori corrected \\
\hline Zugspitze & $6.45[5.84,7.04]$ & $6.07[5.55,6.59]$ & $6.08[5.56,6.60]$ \\
Garmisch & $5.22[4.77,5.65]$ & $5.20[4.74,5.64]$ & $5.19[4.74,5.63]$ \\
\hline
\end{tabular}

series. The trend analysis is performed as described in Gardiner et al. (2008) and Sussmann et al. (2012). In summary, all months with $n>7$ measured spectra are included in the analysis. We then calculate monthly means and subtract the mean annual cycle fitted as a third-order Fourier series from the time series of $\mathrm{XCH}_{4}$ monthly means. Finally a trend is fitted to the deseasonalized time series. The trend uncertainty is determined by means of bootstrap reanalysis.

Table 1 shows the Zugspitze $\mathrm{XCH}_{4}$ trend for the April 2006-March 2015 time interval and its 95\% confidence interval. Due to the major perturbations in the pointing accuracy in the September 2012-September 2014 interval (Fig. 8), the uncorrected Zugspitze trend is no longer consistent with the trend determined from measurements at the nearby Garmisch site. (Note that trend consistency could be shown for the time period before September 2011; see Sussmann et al., 2012.) However, applying mispointing correction restores the consistency of Zugspitze and Garmisch trend results for the whole time series. This holds both for a posteriori-corrected and a priori-corrected results. As outlined in Appendix C, mispointing correction also restores consistency in the bias between the Zugspitze and Garmisch $\mathrm{XCH}_{4}$ monthly means for the phases with differing optical configurations.

\section{Summary and conclusions}

We presented a strategy to determine pointing errors in solar absorption spectrometry due to the sun-tracking device not perfectly targeting at the center of the solar disk. Knowledge of the mispointing allows for subsequent correction of resulting errors in trace gas total column or vertical profile retrievals. Our approach relies on the determination of the Doppler shift of solar spectral lines from measured spectra. However, knowledge of solar line shifts allows a determination of only one component of the mispointing vector on the solar disk, namely the component perpendicular to the solar rotation axis. This does not provide sufficient information to deduce the mispointing component in the zenith direction, which causes errors in trace gas retrievals. We demonstrate, to our knowledge for the first time, a way to overcome this problem. The necessary knowledge of both vector components of the mispointing can be obtained using multiple solar 
line shift measurements at different orientations of the solar rotation axis relative to the zenith direction. Note that this approach relies on the assumption that, within a suitably chosen set of measurements, the change of the mispointing is negligible compared to the magnitude of the mispointing. Therefore, only the systematic component of the mispointing is constrained by this method.

An application of our original mispointing correction strategy was demonstrated via measurements made with the Zugspitze solar FTIR system. We found that a short period of the measurement series (i.e., September 2012September 2014) was notably affected by mispointing problems caused by a non-optimum optical configuration, and a mean vertical mispointing of $-0.063 \pm 0.005^{\circ}$ was derived. As an example for the impact on trace gas retrievals, this mispointing was shown to lead to a mean bias of $-2.82 \pm$ $2.08 \mathrm{ppb}$ in retrieved $\mathrm{XCH}_{4}$ monthly means.

The presented correction strategy relies on the assumption that the change of mispointing within each time bin is negligible compared to its magnitude. It has been outlined in Sect. 4.3 that for the September 2012-September 2014 interval this assumption is well fulfilled at the Zugspitze instrument. For other time intervals and for the Garmisch instrument, the assumption is only poorly fulfilled. However, this is due to the fact that in these cases only minor mispointing occurred, which makes a correction unnecessary. The Zugspitze and Garmisch instruments are likely to be well representative for standard NDACC and TCCON instruments. Therefore for ideally configured NDACC and TCCON systems, a mispointing correction is unlikely to lead to significant accuracy improvements. However, for nonstandard or poorly aligned systems such as the Zugspitze instrument in the 2012-2014 phase, the correction leads to major quality improvements.

The results of the mispointing correction were validated by means of methane trend analysis. The trend derived from uncorrected $\mathrm{XCH}_{4}$ monthly means in the April 2006March 2015 time interval for the Zugspitze site is 6.45 $[5.84,7.04] \mathrm{ppbyr}^{-1}$, inconsistent with the 5.22 [4.77, 5.65 ppb yr $^{-1}$ trend of the nearby Garmisch site. Mispointing correction resolves this inconsistency, resulting in a trend of $6.07[5.55,6.59] \mathrm{ppbyr}^{-1}$ for Zugspitze and 5.20 [4.74, $5.64 \mathrm{ppbyr}^{-1}$ for Garmisch. Furthermore, applying mispointing correction is shown to restore consistency in the bias between the Zugspitze and Garmisch measurements for the phases with differing optical configurations.
In conclusion, the mispointing correction presented in this study is applicable to all kinds of solar absorption spectrometric measurements, i.e., soundings in the mid-infrared and near infrared. First of all, the correction yields benefits for total vertical column retrievals, and we showed an easy-toimplement a posteriori correction, which is simply correcting the retrieved slant column according to the zenith component of the derived mispointing vector. Note that the a posteriori method is designed as a simplified correction approach. However, as outlined in the previous section, the errors that result from the approximations made are negligible for most typical applications. We also presented a more sophisticated correction approach that uses the mispointingcorrected zenith angle as an input to repeated trace gas retrievals. The benefit of this (a priori) correction approach is that also the ray tracing in the forward spectral calculations in the retrieval is performed more accurately according to an improved knowledge of the true zenith angle. While this optional a priori correction approach leads only to minor improvements in terms of the total vertical column result, the a priori correction approach should be preferred if the retrieved target product is the trace gas profile shape (because of the more realistic ray tracing for each atmospheric layer). Finally, the outcome of our paper could be of particular benefit for refining existing records of high-accuracyand-precision greenhouse gas soundings for improved trend analysis and source-sink inversions. 


\section{Appendix A: Orientation of solar rotation axis}

In this section, we outline the calculation of $\alpha_{\text {axis }}$, which is defined as the angle between projections of zenith direction and sun rotation axis on a plane perpendicular to the spectrometer's line of sight. For a sun-viewing instrument, this projection plane corresponds to the plane defined by the daynight separation line on the Earth. The orientation angle $\alpha_{\text {axis }}$ consists of three distinct projections onto the day-night separation plane: the first contribution $\beta$ is the angle between the Earth rotation axis and the zenith direction, the second angle $\gamma$ spans from the ecliptic axis to the Earth rotation axis. The final contribution $\delta$ describes the angle between the sun rotation axis and the ecliptic axis. In total, $\alpha_{\text {axis }}$ is calculated as

$\alpha_{\mathrm{axis}}=\beta+\gamma-\delta$

The calculation of the component $\beta$, ranging from the projections on the day-night separation plane of zenith direction to the Earth rotation axis, is represented in Fig. A1a. The angle $\beta$ has a daily cycle due to the Earth's rotation. The amplitude $\beta_{\max }$ of this daily cycle shows a seasonal variability due to variations in the inclination $\varepsilon$ of the Earth axis relative to the day-night separation plane. The inclination $\varepsilon$ can be calculated as

$\varepsilon=\arcsin \left(\sin \left(\varepsilon_{\max }\right) \cdot \sin (\Upsilon E)\right)$,

where $\Upsilon E$ designates the heliocentric ecliptic longitude of the Earth and $\varepsilon_{\max }=23.43^{\circ}$. This leads to an amplitude of the daily cycle given by

$\beta_{\max }=\arctan \left(\tan \left(90^{\circ}-\right.\right.$ observer latitude $\left.) / \cos \varepsilon\right)$.

The angle $\beta$ can then be expressed in terms of $\beta_{\max }$ and observation time:

$\beta=-\arctan \left(\tan \left(\beta_{\max }\right) \cdot \sin (\right.$ day fraction $\left.\cdot 2 \pi)\right)$,

where day fraction $=$ hours since local noon $/ 24 \mathrm{~h}$. a)

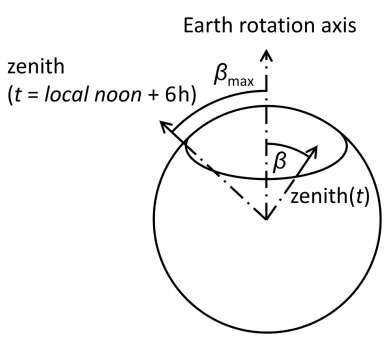

b)

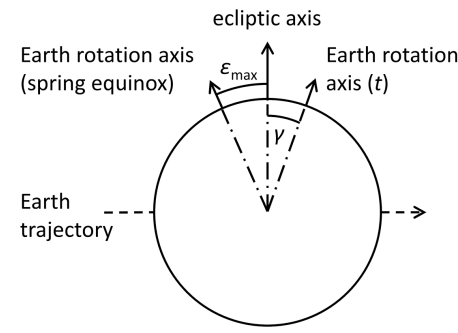

Figure A1. Calculation of sun axis orientation relative to zenith direction. (a) $\beta$ : zenith direction relative to Earth rotation axis. (b) $\gamma$ : Earth rotation axis relative to ecliptic axis. Both subfigures represent projections on the day-night separation plane, direction of view towards the sun.

The second orientation component $\gamma$ is the angle between the Earth rotation axis and the ecliptic axis and has a seasonal cycle due to the inclination of the Earth axis. As shown in Fig. A1b, the amplitude of this seasonal variability is equal to the inclination of the Earth axis relative to the ecliptic axis. Consequently, $\gamma$ can be calculated as

$\gamma=-\arctan \left(\tan \left(\varepsilon_{\max }\right) \cdot \cos (\Upsilon E)\right)$.

The calculation of the third angle component $\delta$, between the sun rotation axis and ecliptic axis, is outlined in Giles (2000). In summary,

$\delta=\arctan (-\cos (\eta-\Omega) \cdot \tan i)$,

where standard values for the constants $i$ and $\Omega$ are $i=$ $7.25^{\circ}, \Omega=73.67^{\circ}+0.013958^{\circ}(t$ (years $\left.)-1850.0\right)$, and $\eta=$ $\Upsilon E+180^{\circ}$. 


\section{Appendix B: Uncertainty estimate of intersection coordinates}

In order to obtain an estimate of the intersection coordinate uncertainty, we adopted the following approach: an estimate of solar line shift uncertainty can be gained from the difference of coincident solar shift measurements in the adjacent $2400<v<3100$ and $2000<v<2650 \mathrm{~cm}^{-1}$ filters. A 15 min coincidence interval was chosen for this calculation. The mean shift difference throughout the April 2006March 2015 interval considered in our study was $3.3 \times 10^{-7}$. Compared to the mean magnitude of solar line shifts of $9.9 \times 10^{-7}$, this corresponds to a mean relative difference of $\sim 33 \%$.

Instead of infinitesimal-width rays as depicted in Fig. 3, the rays $r_{1}$ and $r_{2}$ are assumed to have a width corresponding to the measured solar shift difference. The ray widths $\Delta r_{1}$ and $\Delta r_{2}$ depend on the measured solar line shifts $\Delta s_{1}$ and $\Delta s_{2}$, respectively. Furthermore, due to differential solar rotation, the conversion from shift differences to ray widths depends on the intersection coordinates. The conversion is given by the quotient of the mispointing perpendicular to the solar axis $m_{\mathrm{rad}}$, lin according to Eq. (4) and the measured solar shift $s$ :

$\Delta r=\Delta s \cdot\left(m_{\mathrm{rad}, \mathrm{lin}} / s\right)$.

Due to the finite ray widths $\Delta r_{1}$ and $\Delta r_{2}$, an overlap parallelogram (see Fig. B1) emerges instead of an intersection point. The length of the diagonals of the parallelogram $(e, f)$ can be calculated as follows:

$\Delta \alpha=\alpha_{\text {corr }, 2}-\alpha_{\text {corr }, 1}$,

$a_{1}=\left|\Delta r_{1} / \sin (\Delta \alpha)\right|, a_{2}=\left|\Delta r_{2} / \sin (\Delta \alpha)\right|$,

$e^{2}=a_{1}^{2}+a_{2}^{2}-2 a_{1} a_{2} \cos (\Delta \alpha)$,

$f^{2}=a_{1}^{2}+a_{2}^{2}-2 a_{1} a_{2} \cos \left(180^{\circ}-\Delta \alpha\right)$.

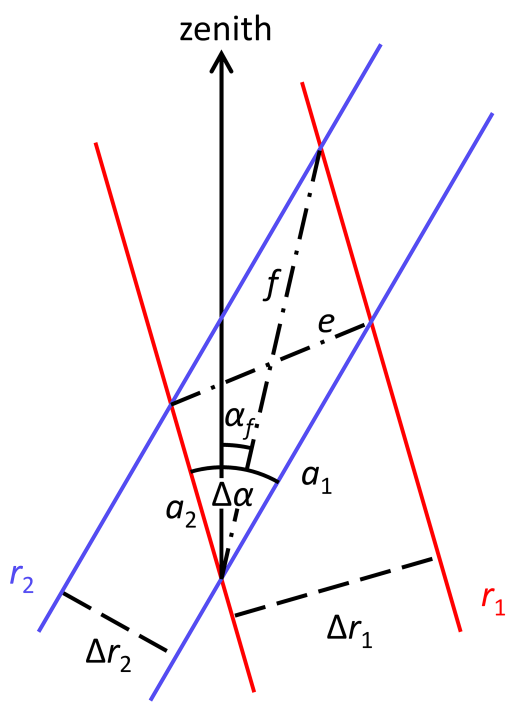

Figure B1. Calculation of mispointing uncertainty.

Finally, the $x$ and $y$ components of the uncertainty estimate, i.e., $\Delta x$ and $\Delta y$, are given by the projection of the overlap parallelogram on the $x$ and $y$ axes, respectively. Note that the $y$ axis is defined to be equivalent to the zenith direction.

$$
\begin{aligned}
& \alpha_{f}=\arccos \left(a_{2}^{2}-a_{1}^{2}-f^{2} /\left(-2 a_{1} f\right)\right)+\alpha_{\mathrm{corr}, 2}, \\
& \Delta x_{e}=\cos \left(\alpha_{f}\right) \cdot e, \Delta x_{f}=\sin \left(\alpha_{f}\right) \cdot f, \\
& \Delta y_{e}=\sin \left(\alpha_{\mathrm{f}}\right) \cdot e, \Delta y_{f}=\cos \left(\alpha_{\mathrm{f}}\right) \cdot f, \\
& \Delta x=\max \left(\Delta x_{e}, \Delta x_{f}\right), \Delta y=\max \left(\Delta y_{e}, \Delta y_{f}\right)
\end{aligned}
$$


Table C1. Bias of $\mathrm{XCH}_{4}$ monthly means between Garmisch and Zugspitze measurements and corresponding $95 \%$ confidence intervals. Results are presented for time intervals with different optical configurations and both a posteriori mispointing-corrected and uncorrected measurements.

\begin{tabular}{lccc}
\hline Time interval & Sep 2010-Aug 2012 & Sep 2012-Oct 2014 & Nov 2014-Mar 2015 \\
\hline Bias uncorrected (\%) & $2.04 \pm 0.18$ & $1.46 \pm 0.27$ & $2.24 \pm 0.37$ \\
Bias a posteriori-corrected $(\%)$ & $1.96 \pm 0.19$ & $1.60 \pm 0.19$ & $2.05 \pm 0.36$ \\
\hline
\end{tabular}

\section{Appendix C: Bias of $\mathrm{XCH}_{4}$ monthly means between Zugspitze and Garmisch measurements}

Due to the lower elevation of the Garmisch site and the decrease of methane concentration with increasing altitude, the $\mathrm{XCH}_{4}$ values measured at this station are generally higher than at the Zugspitze site. Since the mean shape of the methane number density profile is not expected to vary significantly over the time interval considered in this study, the relative bias between the Zugspitze and Garmisch values is expected to be consistent for different optical configurations. Table $\mathrm{C} 1$ shows the mean of $\mathrm{XCH}_{4}$ monthly mean bias over the following time intervals: September 2010 August 2012, i.e., the 2-year period before the optics configuration was changed; September 2012-October 2014, i.e., the approximately 2 -year period with changed optics; and November 2014-March 2015, i.e., the time interval since the setup of the Camtracker system. As visible in Table C1, the bias results are not consistent for the three phases for the uncorrected measurements. However, applying the a posteriori mispointing correction results in consistent bias values for all three phases. 
Acknowledgements. We thank the anonymous referees for their constructive and helpful comments, which led to significant improvements of this manuscript. We furthermore thank H. P. Schmid (KIT/IMK-IFU) for his continual interest in this work and Markus Rettinger (KIT/IMK-IFU) for careful review of all algorithms. Funding by the Bavarian State Ministry of the Environment and Consumer Protection (contracts TLK01U-49581 and VAO-II TP I/01) is gratefully acknowledged. We are grateful for support by the Deutsche Forschungsgemeinschaft and Open Access Publishing Fund of the Karlsruhe Institute of Technology.

The article processing charges for this open-access publication were covered by a Research

Centre of the Helmholtz Association.

Edited by: A. Hofzumahaus

\section{References}

Adrian, G. P., Baumann, M., Blumenstock, T., Fischer, H., Friedle, A., Gerhardt, L., Maucher, G., Oelhaf, H., Scheuerpflug, W., Thomas, P., Trieschmann, O., and Wegner, A.: First results of ground-based FTIR measurements of atmospheric trace gases in North Sweden and Greenland during EASOE, Geophys. Res. Lett., 21, 1343-1346, 1994.

Borsdorff, T. and Sussmann, R.: On seasonality of stratomesospheric CO above midlatitudes: new insight from solar FTIR spectrometry at Zugspitze and Garmisch, Geophys. Res. Lett., 36, L21804, doi:10.1029/2009GL040056, 2009.

Giles, P. M.: Time-Distance Measurements of Large-Scale Flows in the Solar Convection Zone, PhD thesis, Stanford University, CA, USA, 2000.

Gisi, M., Hase, F., Dohe, S., and Blumenstock, T.: Camtracker: a new camera controlled high precision solar tracker system for FTIR-spectrometers, Atmos. Meas. Tech., 4, 47-54, doi:10.5194/amt-4-47-2011, 2011.

Gardiner, T., Forbes, A., de Mazière, M., Vigouroux, C., Mahieu, E., Demoulin, P., Velazco, V., Notholt, J., Blumenstock, T., Hase, F., Kramer, I., Sussmann, R., Stremme, W., Mellqvist, J., Strandberg, A., Ellingsen, K., and Gauss, M.: Trend analysis of greenhouse gases over Europe measured by a network of ground-based remote FTIR instruments, Atmos. Chem. Phys., 8, 6719-6727, doi:10.5194/acp-8-6719-2008, 2008.

Hase, F.: Inversion von Spurengasprofilen aus hochaufgelösten bodengebundenen FTIR-Messungen in Absorption, $\mathrm{PhD}$ thesis, FZK Report No. 6512, Karlsruhe Institute of Technology, Germany, available at: http://www.imk-asf.kit.edu/downloads/bod/ disshase.pdf (last access: 17 June 2015), 2000.

Hase, F., Hannigan, J. W., Coffey, M. T., Goldman, A., Höpfner, M., Jones, N. B., Rinsland, C. P., and Wood, S. W.: Intercomparison of retrieval codes used for the analysis of high-resolution, ground-based FTIR measurements, J. Quant. Spectrosc. Ra., 87, 25-52, doi:10.1016/j.jqsrt.2003.12.008, 2004.

Kasten, F., and Young, A. T.: Revised optical air mass tables and approximation formula, Appl. Opt. 28, 4735-4738, doi:10.1364/AO.28.004735, 1989.

Kohlhepp, R., Ruhnke, R., Chipperfield, M. P., De Mazière, M., Notholt, J., Barthlott, S., Batchelor, R. L., Blatherwick, R. D.,
Blumenstock, Th., Coffey, M. T., Demoulin, P., Fast, H., Feng, W., Goldman, A., Griffith, D. W. T., Hamann, K., Hannigan, J. W., Hase, F., Jones, N. B., Kagawa, A., Kaiser, I., Kasai, Y., Kirner, O., Kouker, W., Lindenmaier, R., Mahieu, E., Mittermeier, R. L., Monge-Sanz, B., Morino, I., Murata, I., Nakajima, H., Palm, M., Paton-Walsh, C., Raffalski, U., Reddmann, Th., Rettinger, M., Rinsland, C. P., Rozanov, E., Schneider, M., Senten, C., Servais, C., Sinnhuber, B.-M., Smale, D., Strong, K., Sussmann, R., Taylor, J. R., Vanhaelewyn, G., Warneke, T., Whaley, C., Wiehle, M., and Wood, S. W.: Observed and simulated time evolution of $\mathrm{HCl}, \mathrm{ClONO}_{2}$, and $\mathrm{HF}$ total column abundances, Atmos. Chem. Phys., 12, 3527-3556, doi:10.5194/acp-12-3527-2012, 2012.

Liu, X., Blatherwick, R. D., Murcray, F. J., Keys, J. G., and Solomon, S.: Measurements and model calculations of $\mathrm{HCl}$ column amounts and related parameters over McMurdo during the austral spring in 1989, J. Geophys. Res., 97, 20795-20804, doi:10.1029/92JD02435, 1992.

Mahieu, E., Chipperfield, M. P., Notholt, J., Reddmann, T., Anderson, J., Bernath, P. F., Blumenstock, T., Coffey, M. T., Dhomse, S. S., Feng, W., Franco, B., Froidevaux, L., Griffith, D. W. T., Hannigan, J. W., Hase, F., Hossaini, R., Jones, N. B., Morino, I., Murata, I., Nakajima, H., Palm, M., Paton-Walsh, C., Russell III, J. M., Schneider, M., Servais, C., Smale, D., and Walker, K. A.: Recent Northern Hemisphere stratospheric $\mathrm{HCl}$ increase due to atmospheric circulation changes, Nature, 515, 104-107, doi:10.1038/nature13857, 2014.

Notholt, J., and Schrems, O.: Shipborne FT-IR Measurements of Atmospheric Trace Gases on a South $\left(33^{\circ} \mathrm{S}\right)$ to North $\left(53^{\circ} \mathrm{N}\right)$ Atlantic Traverse, Appl. Spectrosc., 49, 1525-1527, 1995.

Ostler, A., Sussmann, R., Rettinger, M., Deutscher, N. M., Dohe, S., Hase, F., Jones, N., Palm, M., and Sinnhuber, B.-M.: Multistation intercomparison of column-averaged methane from NDACC and TCCON: impact of dynamical variability, Atmos. Meas. Tech., 7, 4081-4101, doi:10.5194/amt-7-4081-2014, 2014.

Ostler, A., Sussmann, R., Patra, P. K., Wennberg, P. O., Deutscher, N. M., Grith, D. W. T., Blumenstock, T., Hase, F., Kivi, R., Warneke, T., Wang, Z., de Mazière, M., Robinson, J., and Ohyama, H.: The imprint of stratospheric transport on columnaveraged methane, Atmos. Chem. Phys., submitted, 2015.

Palm, M., Melsheimer, C., Noël, S., Heise, S., Notholt, J., Burrows, J., and Schrems, O.: Integrated water vapor above $\mathrm{Ny}$ Ålesund, Spitsbergen: a multi-sensor intercomparison, Atmos. Chem. Phys., 10, 1215-1226, doi:10.5194/acp-10-1215-2010, 2010.

Pougatchev, N. S. and Rinsland, C. P.: Spectroscopic study of the seasonal variation of carbon monoxide vertical distribution above Kitt Peak, J. Geophys. Res., 100, 1409-1416, doi:10.1029/94JD02387, 1995.

Pougatchev, N. S., Connor, B. J., Jones, N. B., and Rinsland, C. P.: Validation of ozone profile retrievals from infrared ground-based solar spectra, Geophys. Res. Lett., 23, 1637-1640, doi:10.1029/96GL01501, 1996.

Rinsland, C. P., Connor, B. J., Jones, N. B., Boyd, I., Matthews, W. A., Goldman, A., Murcray, F. J., Murcray, D. G., David, S. J., and Pougatchev, N. S.: Comparison of infrared and Dobson total ozone columns measured from Lauder, New Zealand, Geophys. Res. Lett., 23, 1025-1028, doi:10.1029/96GL00708, 1996. 
Rinsland, C. P., Mahieu, E., Zander, R., Jones, N. B., Chipperfield, M. P., Goldman, A., Anderson, J., Russell, J. M., Demoulin, P., Notholt, J., Toon, G. C., Blavier, J.-F., Sen, B., Sussmann, R., Wood, S. W., Meier, A., Griffith, D. W. T., Chiou, L. S., Murcray, F. J., Stephen, T. M., Hase, F., Mikuteit, S., Schulz, A., and Blumenstock, T.: Long-term trends of inorganic chlorine from ground-based infrared solar spectra: past increases and evidence for stabilization, J. Geophys. Res., 108, 4252-4272, doi:10.1029/2002JD003001, 2003.

Schneider, M. and Hase, F.: Technical Note: Recipe for monitoring of total ozone with a precision of around 1 DU applying midinfrared solar absorption spectra, Atmos. Chem. Phys., 8, 63-71, doi:10.5194/acp-8-63-2008, 2008.

Schneider, M., Hase, F., and Blumenstock, T.: Water vapour profiles by ground-based FTIR spectroscopy: study for an optimised retrieval and its validation, Atmos. Chem. Phys., 6, 811-830, doi:10.5194/acp-6-811-2006, 2006.

Sepúlveda, E., Schneider, M., Hase, F., García, O. E., GomezPelaez, A., Dohe, S., Blumenstock, T., and Guerra, J. C.: Longterm validation of tropospheric column-averaged $\mathrm{CH}_{4}$ mole fractions obtained by mid-infrared ground-based FTIR spectrometry, Atmos. Meas. Tech., 5, 1425-1441, doi:10.5194/amt-5-14252012, 2012.

Snodgrass, H. B. and Ulrich, R. K.: Rotation of Doppler features in the solar photosphere, Astrophys. J., 351, 309-316, doi:10.1086/168467, 1990.

Sussmann, R. and Schäfer, K.: Infrared spectroscopy of tropospheric trace gases: combined analysis of horizontal and vertical column abundances, Appl. Optics, 36, 735-741, doi:10.1364/AO.36.000735, 1997.

Sussmann, R., Borsdorff, T., Rettinger, M., Camy-Peyret, C., Demoulin, P., Duchatelet, P., Mahieu, E., and Servais, C.: Technical Note: Harmonized retrieval of column-integrated atmospheric water vapor from the FTIR network - first examples for longterm records and station trends, Atmos. Chem. Phys., 9, 89878999, doi:10.5194/acp-9-8987-2009, 2009.

Sussmann, R., Forster, F., Rettinger, M., and Jones, N.: Strategy for high-accuracy-and-precision retrieval of atmospheric methane from the mid-infrared FTIR network, Atmos. Meas. Tech., 4, 1943-1964, doi:10.5194/amt-4-1943-2011, 2011.

Sussmann, R., Forster, F., Rettinger, M., and Bousquet, P.: Renewed methane increase for five years (2007-2011) observed by solar FTIR spectrometry, Atmos. Chem. Phys., 12, 4885-4891, doi:10.5194/acp-12-4885-2012, 2012.

Sussmann, R., Ostler, A., Forster, F., Rettinger, M., Deutscher, N. M., Griffith, D. W. T., Hannigan, J. W., Jones, N., and Patra, P. K.: First intercalibration of column-averaged methane from the Total Carbon Column Observing Network and the Network for the Detection of Atmospheric Composition Change, Atmos. Meas. Tech., 6, 397-418, doi:10.5194/amt-6397-2013, 2013.
Toon, G., Blavier, J.-F., Washenfelder, R., Wunch, D., KeppelAleks, G., Wennberg, P., Connor, B., Sherlock, V., Griffith, D., Deutscher, N., and Notholt, J.: Total Column Carbon Observing Network (TCCON), available at: http://www.tccon.caltech.edu/ publications/OSA_FTS_Meeting_20090323.pdf (last access: 10 September 2015), 2009.

Vigouroux, C., De Mazière, M., Demoulin, P., Servais, C., Hase, F., Blumenstock, T., Kramer, I., Schneider, M., Mellqvist, J., Strandberg, A., Velazco, V., Notholt, J., Sussmann, R., Stremme, W., Rockmann, A., Gardiner, T., Coleman, M., and Woods, P.: Evaluation of tropospheric and stratospheric ozone trends over Western Europe from ground-based FTIR network observations, Atmos. Chem. Phys., 8, 6865-6886, doi:10.5194/acp-8-6865-2008, 2008.

Washenfelder, R. A., Wennberg, P. O., and Toon, G. C.: Tropospheric methane retrieved from ground-based near-IR solar absorption spectra, Geophys. Res. Lett., 30, 2226, doi:10.1029/2003GL017969, 2003.

Washenfelder, R. A., Toon, G. C., Blavier, J.-F., Yang, Z., Allen, N. T., Wennberg, P. O., Vay, S. A., Matross, D. M., and Daube, B. C.: Carbon dioxide column abundances at the Wisconsin Tall Tower site, J. Geophys. Res., 111, D22305, doi:10.1029/2006JD007154, 2006.

Wunch, D., Toon, G. C., Blavier, J.-F. L., Washenfelder, R. A., Notholt, J., Connor, B. J., Griffith, D. W. T., Sherlock, V., and Wennberg, P. O.: The Total Carbon Column Observing Network, Philos. T. Roy. Soc. A, 369, 2087-2112, doi:10.1098/rsta.2010.0240, 2011.

Zander, R., Roland, G., Delbouille, L. Sauval, A., Farmer, C. B., and Norton, R. H.: Monitoring of the integrated column of hydrogen fluoride above the Jungfraujoch Station since 1977 - the $\mathrm{HF} / \mathrm{HCl}$ column ratio, J. Atmos. Chem., 5, 385-394, doi:10.1007/BF00113901, 1987.

Zander, R., Ehhalt, D. H., Rinsland, C. P.,Schmidt, U., Mahieu, E., Rudolph, J., Demoulin, P., Roland, G., Delbouille, L., and Sauval, A. J.: Secular trend and seasonal variability of the column abundance of $\mathrm{N}_{2} \mathrm{O}$ above the Jungfraujoch station determined from IR solar spectra, J. Geophys. Res., 99, 16745-16756, doi:10.1029/94JD01030, 1994. 